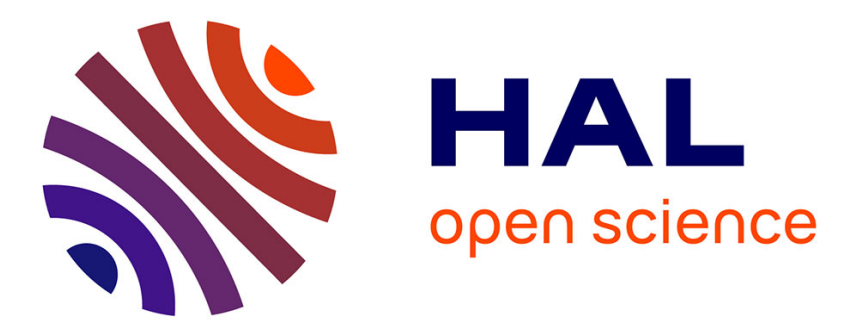

\title{
Survey of Saturn electrostatic cyclotron harmonic wave intensity
}

J. Menietti, T. F. Averkamp, W. Kurth, S.-Y. Ye, D. Gurnett, B. Cecconi

\section{To cite this version:}

J. Menietti, T. F. Averkamp, W. Kurth, S.-Y. Ye, D. Gurnett, et al.. Survey of Saturn electrostatic cyclotron harmonic wave intensity. Journal of Geophysical Research Space Physics, 2017, 122 (8), pp.8214-8227. 10.1002/2017JA023929 . obspm-02194394

\section{HAL Id: obspm-02194394 https://hal-obspm.ccsd.cnrs.fr/obspm-02194394}

Submitted on 18 Nov 2021

HAL is a multi-disciplinary open access archive for the deposit and dissemination of scientific research documents, whether they are published or not. The documents may come from teaching and research institutions in France or abroad, or from public or private research centers.
L'archive ouverte pluridisciplinaire HAL, est destinée au dépôt et à la diffusion de documents scientifiques de niveau recherche, publiés ou non, émanant des établissements d'enseignement et de recherche français ou étrangers, des laboratoires publics ou privés.

$$
\text { Copyright }
$$




\section{Journal of Geophysical Research: Space Physics}

\author{
RESEARCH ARTICLE \\ 10.1002/2017JA023929 \\ Key Points: \\ - Saturn ECH wave intensity is binned in \\ $\mathrm{L}$, latitude, and local time \\ - Median distributions are much more \\ ordered than mean distributions \\ - Plasma injection and anomalous \\ emission regions can have significant \\ ECH wave intensity
}

Correspondence to:

J. D. Menietti,

john-menietti@uiowa.edu

\section{Citation:}

Menietti, J. D., T. F. Averkamp,

W. S. Kurth, S.-Y. Ye, D. A. Gurnett, and

B. Cecconi (2017), Survey of Saturn

electrostatic cyclotron harmonic wave

intensity, J. Geophys. Res. Space Physics,

122, 8214-8227, doi:10.1002/

$2017 J A 023929$.

Received 20 JAN 2017

Accepted 28 JUN 2017

Accepted article online 5 JUL 2017

Published online 9 AUG 2017
@2017. American Geophysical Union. All Rights Reserved.

\section{Survey of Saturn electrostatic cyclotron harmonic wave intensity}

\author{
J. D. Menietti' ${ }^{1}$, T. F. Averkamp ${ }^{1}$ D, W. S. Kurth ${ }^{1}$ (D), S.-Y. Ye' ${ }^{1}$, D. A. Gurnett ${ }^{1}(\mathbb{D}$, \\ and B. Cecconi ${ }^{2}$ iD \\ ${ }^{1}$ Department of Physics and Astronomy, University of lowa, lowa City, lowa, USA, ${ }^{2}$ LESIA, Observatoire de Paris, CNRS, PSL \\ Research University, UMPC, Université Denis Diderot, Meudon, France
}

\begin{abstract}
We conduct a survey of electrostatic electron cyclotron harmonic (ECH) emissions observed at Saturn by the radio and plasma wave science investigation on board the Cassini spacecraft. These emissions are known to be effective at interacting with electrons in the terrestrial inner magnetosphere, producing electron scattering into the loss cone and acceleration (cf. Horne and Thorne, 2000; Thorne et al., 2010). At Saturn ECH emission occurs with high probability and at strong intensity near the magnetic equator, outside the Enceladus torus in the range $\sim 5<\mathrm{L}<10$. Inside the inner boundary of the torus, $\mathrm{ECH}$ emissions are also observed near the equator and at higher latitude. Intensity levels of ECH emission are comparable to those observed at Earth, higher than Saturn chorus and Z-mode emission, and are likely to scatter electrons into the loss cone as at Earth. ECH waves are particularly intense and extend to higher harmonics within some plasma injection regions. We present results for a survey of over 8 years of Saturn data for fundamental and up to three harmonics of $f_{c e}$, the electron cyclotron frequency.
\end{abstract}

\section{Introduction}

Chorus emission at Earth is known to be a significant source of electron energization [cf. Thorne et al., 2010, and references therein; Reeves et al., 2013], and has recently been studied using global models at Jupiter and Saturn [Shprits et al., 2012; Woodfield et al., 2014]. Global modeling of wave particle interactions involves stochastic, diffusive scattering of electrons by chorus, $Z$ mode, electron cyclotron harmonic (ECH), and other waves. These models require the diffusion coefficient for pitch angle and momentum scattering of the electrons due to resonant interactions of the waves [Horne and Thorne, 2000; Glauert and Horne, 2005]. Necessary for such global models are the global distributions of electron and wave intensity [cf. Shprits et al., 2009, 2012; Horne et al., 2008; Woodfield et al., 2014]. The distribution of wave intensity as a function of spatial parameters ( $L$ shell, latitude, and magnetic local time) as well as frequency is necessary. These distributions are then used to obtain functional forms of the wave intensity for use in global modeling codes. While wave particle interactions are now believed to be a significant source of electron acceleration and loss of radiation belt electrons at Earth, the importance of such interactions at Saturn is an ongoing study. A survey of Saturn chorus and Z-mode emission intensity has been published in Menietti et al. [2014, 2015]; however, ECH emission survey studies at Saturn remain to be reported.

Electron cyclotron harmonics are narrowband electrostatic Bernstein modes at approximately $(n+1 / 2) f_{c e}$ and are nearly ubiquitous in planetary magnetospheres. Field strengths at Earth can be quite strong ( 1 mV/m) [Koons and Roderer, 1990; Kurth et al., 1980]. The source of free energy required for wave growth can be loss cones, temperature anisotropies, or a positive slope in the electron perpendicular velocity distribution [Ashour-Abdalla and Kennel, 1978]. At Earth ECH emissions can resonate with plasma sheet electrons causing precipitation of electrons with energies less than a few keV [Thorne et al., 2010].

Gurnett et al. [2005] and Moncuquet et al. [2005] have reported intense ECH and upper hybrid emission in the Saturn magnetosphere [cf. Gurnett et al., 2005, Figure 3]. These emissions have for years been known to scatter electrons in the terrestrial magnetosphere into the loss cone and likely provide at least one source of the diffuse aurora [cf. Horne and Thorne, 2000; Horne et al., 2003; Thorne et al., 2010]. Surveys of ECH at Earth have shown a dominant source near the equator (local minimum field strength) outside the plasmapause in the range $4<\mathrm{L}<7$ [Meredith et al., 2009; Ni et al., 2011]. ECH emission at Saturn is observed in plasma injection regions as reported by Hospodarsky et al. [2008]. Injection regions are known to carry warm, tenuous plasma from larger radial distances inward and cooler, more dense plasma outward in the Saturn magnetosphere, 
driven by the plasma interchange instability [cf. Hill et al., 1981, 2005; Pontius et al., 1986]. Menietti et al. [2008] and Tao et al. [2010] have conducted initial studies of wave growth based on the electron distribution observed within these regions. These emissions are typically more intense than in other quiescent regions of Saturn's magnetosphere, with an unusually high number of harmonics [cf. Menietti et al., 2012, Figure 10], probably due to the increase in the ratio of warm to cold plasma. However, plasma injection regions occur intermittently and are typically observed for relatively brief times. These events are included in this study, but only up to $9 / 2 f_{c e}$, because it is difficult in a computer survey to distinguish each injection region from $\mathrm{ECH}$ emission. Another important source of intense emission seen in the same frequency range of ECH waves is the narrowband source regions discussed by Ye et al. [2009]. Narrowband source regions are associated with intense upper hybrid resonance emission, possibly due to special conditions such as when the upper hybrid frequency, $f_{\mathrm{uh}}$, is close to $2 f_{c e}$ or $3 f_{c e}$ [Yoon et al., 1998; Menietti et al., 2009]. These regions appear as intensifications of the electrostatic upper hybrid emission and are source regions of electromagnetic narrowband emission in both the ordinary and $Z$ mode [cf. Menietti et al., 2009, Figure 1c]. These emissions therefore overlap the frequency range of ECH emission. Such emission is difficult to distinguish from ECH emission without personal examination of a spectrogram. Plasma injections and anomalous emission regions such as narrowband emission source regions are not the specific focus of this investigation, but we will examine the impact of the most intense of these emissions later in section 6.

In the present work we report a survey of ECH observations at Saturn in the approximate range $2.5 R_{s}<r<10$ $R_{s}$, over a large range of $f_{c e}$ and for $f_{p e} / f_{c e}$ both less than and greater than 1 . We find that ECH emission is quite common in the Saturn equatorial plane with largest intensities seen inside plasma injection or anomalous emission regions.

\section{Methodology}

The general scheme for conducting the computer survey of perikrone passes is to introduce spatial bins in magnetic $L$ shell $(L)$, magnetic local time $(M L T)$, and magnetic latitude $(\lambda)$. A computer algorithm is used to conduct a search for ECH emission in bands of width $f_{c e}$ over the range $f_{c e}<f<5 f_{c e}$. We define the harmonic band $\Delta f_{n}=n f_{c e}$ where $n$ is an integer. There can be many instrumental frequency channels within each $\Delta f_{n}$ at each time step, $\Delta \tau_{i}$. The wave intensities are proportional to $P=E^{2}\left(\mathrm{~V}^{2} / \mathrm{m}^{2}\right)$. From the measured electric spectral density, $\chi(f)$, measured in $\mathrm{V}^{2} /\left(\mathrm{m}^{2}-\mathrm{Hz}\right)$ over a range of frequencies, we determine $E^{2}\left(\Delta f_{n}\right)$ (measured in $\mathrm{V}^{2} / \mathrm{m}^{2}$ ), by integration over the frequency channels within $\Delta f_{n}$ for a time step, $\Delta \tau_{i}(1 \mathrm{~min})$. The sum of these integrations over $\Delta \tau_{i}$ is called $P_{n i}$ where subscript " $n$ " refers to the frequency band. The spacecraft position $\left(r, \lambda\right.$, and MLT) at each time step, $\Delta \tau_{i}$ is always recorded. We then calculate both the mean and median value of all $P_{n i}$ within any chosen spatial bin $(\Delta r, \Delta \lambda$, and $\Delta \mathrm{MLT})$ during the total integration time $\left(T_{\text {total }}=\Sigma_{i} \Delta \tau_{i}\right)$. The definitions of mean and median of a sample of points can be found, for instance, in Bevington [1969]. As an example, for a particular $\Delta \mathrm{L}$, by calculating the mean and median for all values of MLT, and all values of $\lambda$ over $T_{\text {total }}$ we can obtain $P_{n}(\mathrm{~L})$, and similarly for $P_{n}(\lambda)$. In this paper the "number of points" refers to the number of values of $P_{n i}$ for a specified bin.

The spectral density values were obtained at Saturn from the Radio and Plasma Wave Science (RPWS) instrument [Gurnett et al., 2004]. This complex instrument includes three low-time-resolution receivers, for low-, medium-, and high-frequency measurements: LFR (1-26 Hz), MFR (24 Hz-12 kHz), and HFR (3.5 kHz-16 MHz). For this study we have used the MFR and HFR data. No data satisfying the constraints for ECH emission were obtained at the low frequencies of the LFR. There are three approximately orthogonal electric antennas $(10 \mathrm{~m})$ and three orthogonal search coils for magnetic measurements. The MFR usually toggles between the $E_{x}$ and $B_{z}$ antennas every $32 \mathrm{~s}$ (16 s each), $\Delta f / f \sim 7 \%$. For this study only the electric antenna data are retrieved for the search of electrostatic cyclotron harmonic emissions. The HFR receiver can be operated in either the two-antenna (dipole-monopole) mode or the three-antenna (direction finding) mode (see Cecconi and Zarka [2005] for more details). The HFR consists of two sets of four analog receivers. Three of four analog receivers, used in this study, have fixed-frequency filters that together cover the frequency range 3.5 to $319 \mathrm{kHz}$. Digital spectral analysis within each of the three filter bands provides 8,16 , or 32 logarithmically spaced frequency channels, depending on mode, providing spectral resolutions of 20,10 , or $5 \%$, respectively [Gurnett et al., 2004]. 


\section{AGU Journal of Geophysical Research: Space Physics}

a

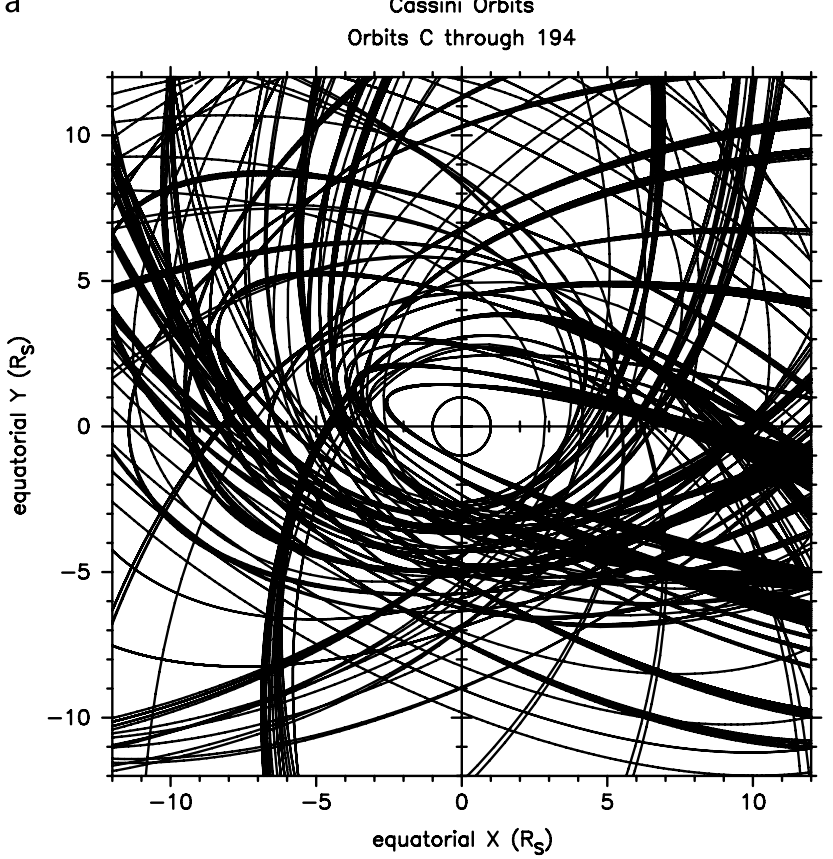

A-D16-165

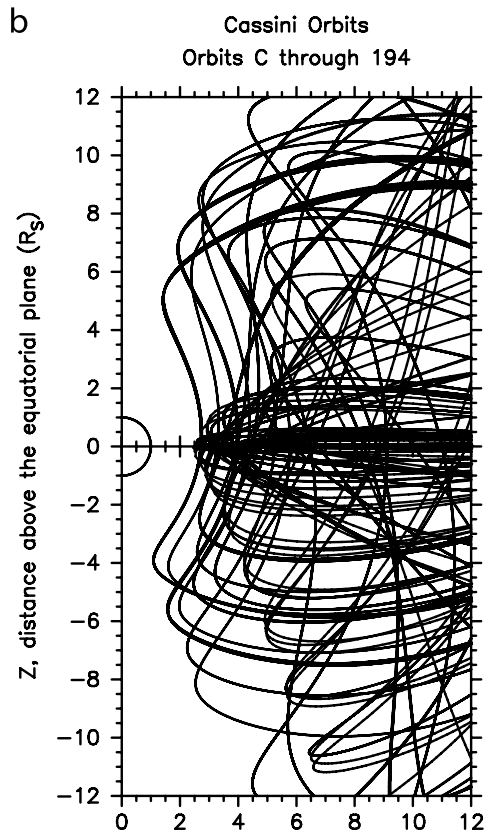

$\rho$, distance from Saturn projected on the equatorial plane $\left(R_{S}\right)$

Figure 1. Cassini orbits for the ECH emission survey. (a) Equatorial ( $x-y)$ plane. (b) Meridian ( $\rho-z)$ plane.

A background spectral density is computed for every frequency channel measured by the RPWS instrument. At each frequency all the spectral densities within a chosen time interval are sorted in amplitude from smallest to largest. The time interval is generally about 1 day or the time for a perikrone (or periapsis) pass. Based on past analysis of occurrence rates versus spectral density for the RPWS receivers, the lowest $30 \%$ of the data are deemed below the noise level. Additional information regarding the receivers can be found in Gurnett et al. [2004].

Apparent wave polarization of the observations is determined using the Meudon Cassini RPWS HFR database (http://www.lesia.obspm.fr/kronos/data) which contains, among many other parameters, the apparent Stokes parameters necessary to determine the apparent linear and circular polarization of the two-antenna and three-antenna mode HFR data [cf. Cecconi and Zarka, 2005; Fischer et al., 2009; Ye et al., 2010]. Apparent polarization is distinguished from real polarization which requires that the electric field wave components lie in a plane perpendicular to the wave vector. Because the direction of the wave vector is not always known, the apparent polarization is defined to be given by the wave electric field components measured by two orthogonal antennas in the antenna plane [cf. Ye et al., 2010; Fischer et al., 2009].

The radio effective antenna lengths used are given in Zarka et al. [2004]. If the observed emission is circularly polarized with signal-to-noise ratio (SNR) larger than background, this emission is rejected for not being electrostatic. The criteria required for linear polarization is $\sqrt{q^{2}+u^{2}}>0.5$ and $|v|<0.3$, where $q, u$, and $v$ are the normalized apparent Stokes parameters [Ye et al., 2010].

\section{Models and Data Constraints}

$\mathrm{ECH}$ emissions are measured at the fundamental and harmonics up to the local upper hybrid frequency, $f_{\mathrm{uh}}=\sqrt{f_{p e}^{2}+f_{c e}^{2}}$, where $f_{p e}$ is the local plasma density. The upper hybrid resonance frequency has been digitally catalogued for much of the Cassini mission period considered in this study [cf. Persoon et al., 2013], and, when available, the values are interpolated during the calculations of our analysis. The uncertainty of the measurement of the upper hybrid frequency is typically the width in frequency of the resonance emission line. The spectral resolutions of the MFR and HFR are stated above. However, when the upper hybrid resonance is not observed or uncertain, we use interpolated values of the density obtained from the Langmuir Probe, 
A-D15-023-3

a 2005-09-23 (266) 02:00

2005-09-24 (267) 18:00
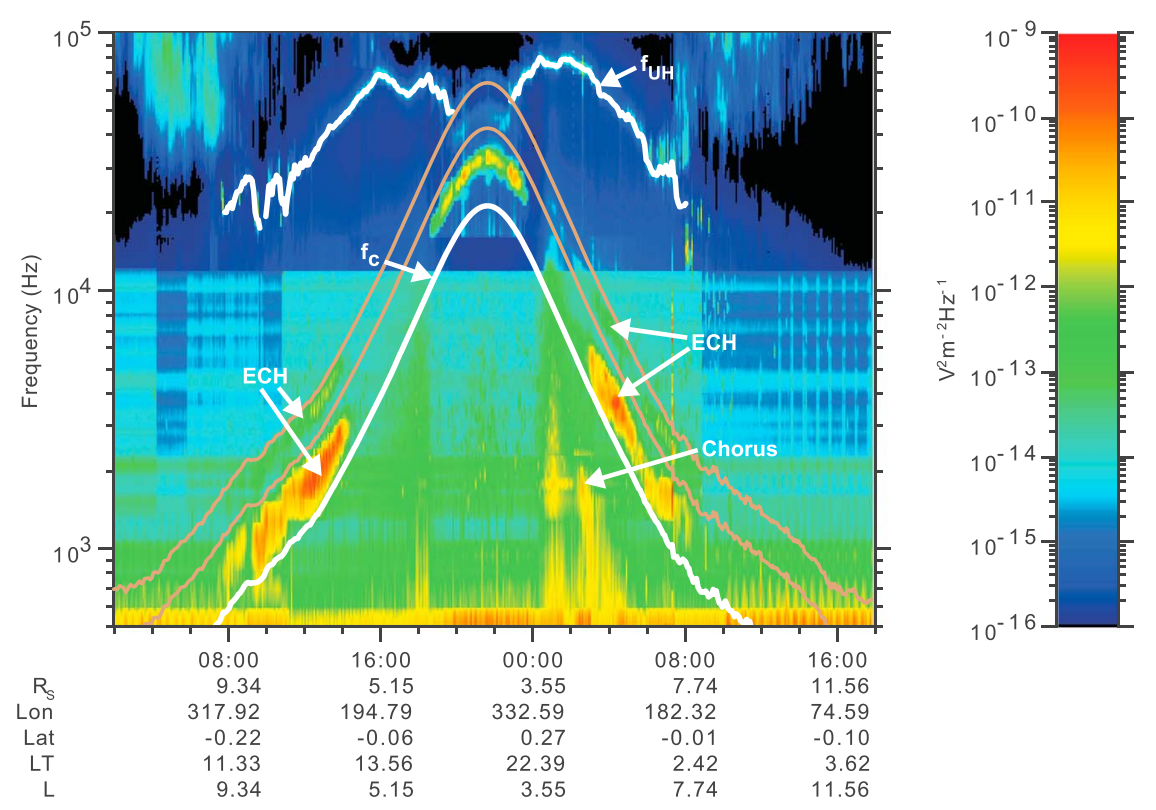

Orbit 15

b
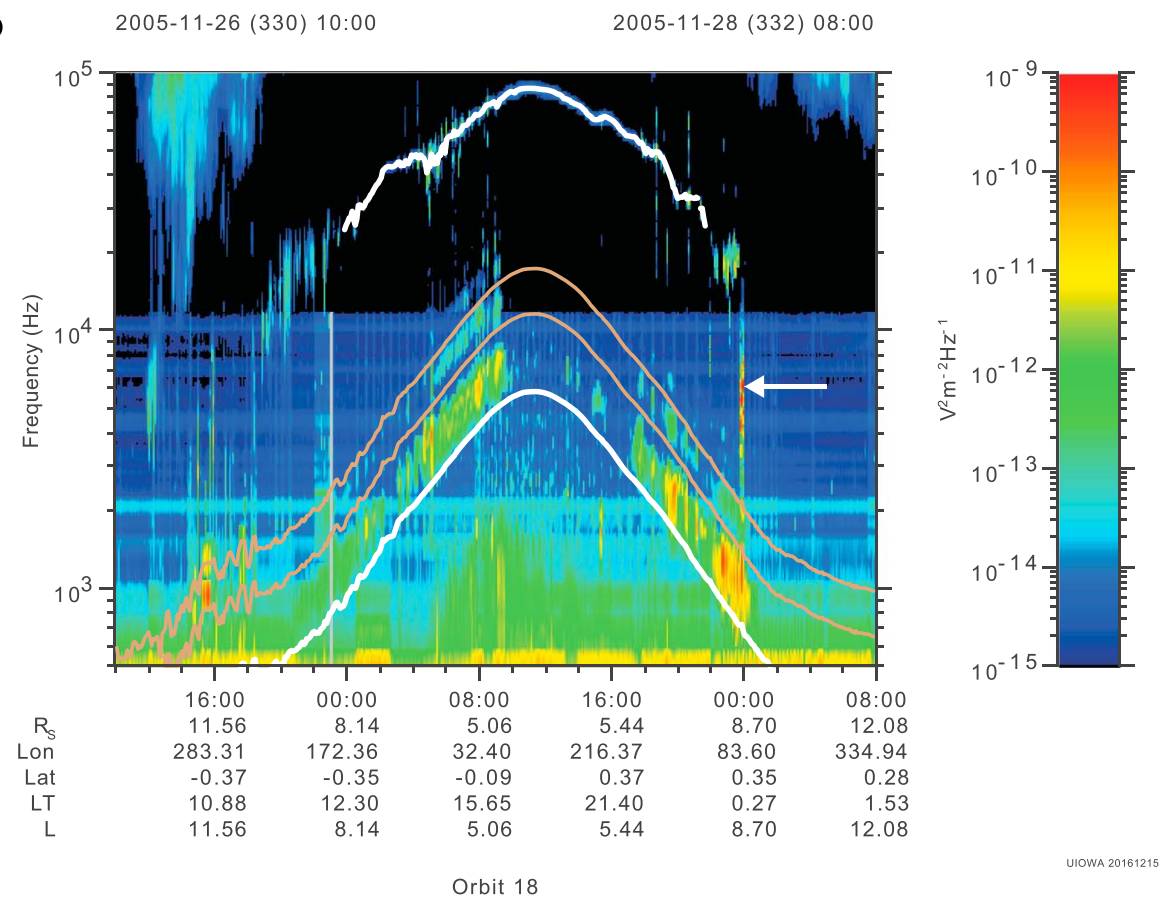

Figure 2. (a) A frequency-time spectrogram with calibrated spectral density color-coded, for an equatorial orbit for times $2005 / 266$ 02:00 to 2005/267 18:00. The dominant emission on the spectrogram is the ECH emission in the fundamental (3/2) and two weaker harmonics $(5 / 2,7 / 2)$. (b) Another perigee pass of a Cassini equatorial orbit with a perigee that lies in denser plasma than the pass of Figure 2a. Note the anomalous emission region indicated by the arrow.

an instrument on board Cassini and part of the RPWS [Wahlund et al., 2005; Morooka et al., 2009]. Density measurements from the Langmuir Probe are reported to agree well with those of Persoon et al. [2009] [cf. Morooka et al., 2009]. Finally, if these values are not available, we use the Saturn plasma density model of Persoon et al. [2013] for Saturn's inner magnetosphere. Persoon et al. [2015] have noted a 


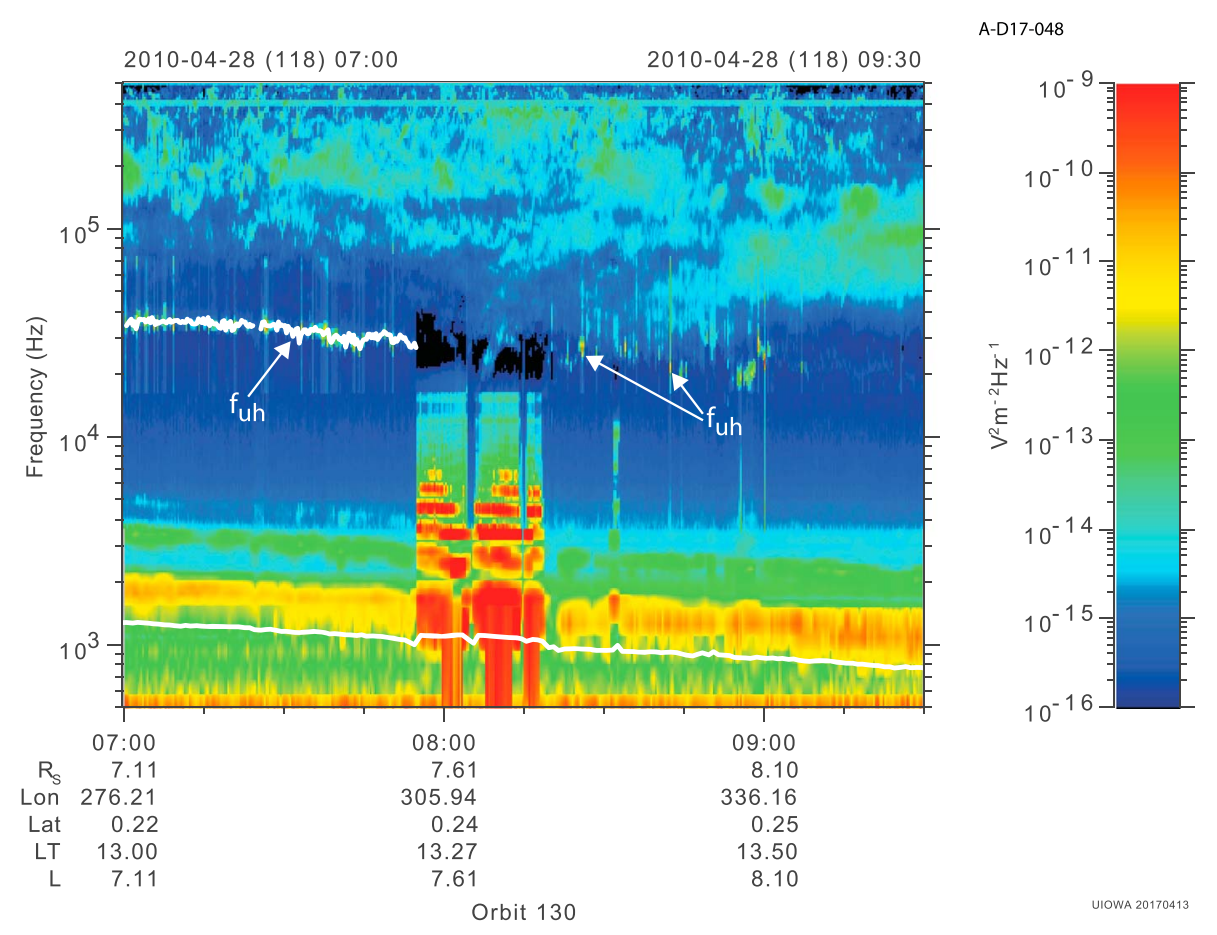

Figure 3. A higher resolution plot of anomalous emission, likely a plasma injection region, showing isolated intense harmonics for the time period of 07:54-08:19. The cyclotron frequency is also enhanced during this time.

seasonal dependence of the plasma density in the region between Saturn's A ring and Enceladus orbit which is not taken into account in this study. The dependence is confined to the equatorial region inside Enceladus orbit, which is usually not a part of our investigation. For the survey we found the following distribution of times each method was utilized: upper hybrid density-31\%, Langmuir Probe density-34\%, model density- $13 \%$, and no value was available $22 \%$ of the time. If no measurement of $f_{\text {uh }}$ is possible, harmonics of $\mathrm{ECH}$ are measured up to $9 / 2 f_{c e}$ maximum. The electron cyclotron frequency used in this study is the local (spacecraft) value and is calculated from $1 \mathrm{~min}$ averages of the fluxgate magnetometer measurements [Dougherty et al., 2004]. To avoid the E-ring (generated by Enceladus) when Enceladus is not nearby, we omit observations within $2500 \mathrm{~km}$ of the equator and in the radial range 3.75 $R_{s}<r<4.4 R_{s}$. During an Enceladus flyby orbit we increase the distance from $2500 \mathrm{~km}$ to $3500 \mathrm{~km}$.

To avoid spacecraft and instrumental interference as well as natural wave interference, in the computer search for ECH emission, we place constraints on the data analyzed in this survey, which are summarized as the following:

1. Cassini orbits with $r<11 R_{s}$ at the equator and latitude, $\lambda<40^{\circ}$ from 2005 day 015 to 2013 day 141.

2. Background spectral density, $\chi_{\mathrm{min}}$, is determined dynamically for each periapsis.

3. $f_{\min }=f_{c e}, f_{\max }=f_{\text {uh }}$ or $f_{\max }=5 f_{c e}$, whichever is lower.

4. Avoid observations near ring plane crossings of the high density E-ring, where dust impact noise is intense.

5. Remove electromagnetic emission with circular polarization above the prescribed signal-to-noise ratio.

The data for the Saturn survey were obtained over approximately 7.5 years from 15 January 2005 to 8 July 2013. In Figure 1 we display plots of the orbits in the equatorial $(x-y)$ and $(\rho-z)$ planes, confined to $12 R_{s}$ $\left(R_{s}=\right.$ Saturn radius). These plots together provide a sense of the overall spacecraft coverage. All of these orbits were surveyed for ECH emission satisfying the above constraints.

\section{Saturn ECH Intensity Surveys}

Figure $2 \mathrm{a}$ is a frequency-time spectrogram with calibrated spectral density color-coded. The brown curves are harmonics of $f_{c e}$. The data shown are for a perikrone pass of the Cassini spacecraft for an equatorial orbit for 

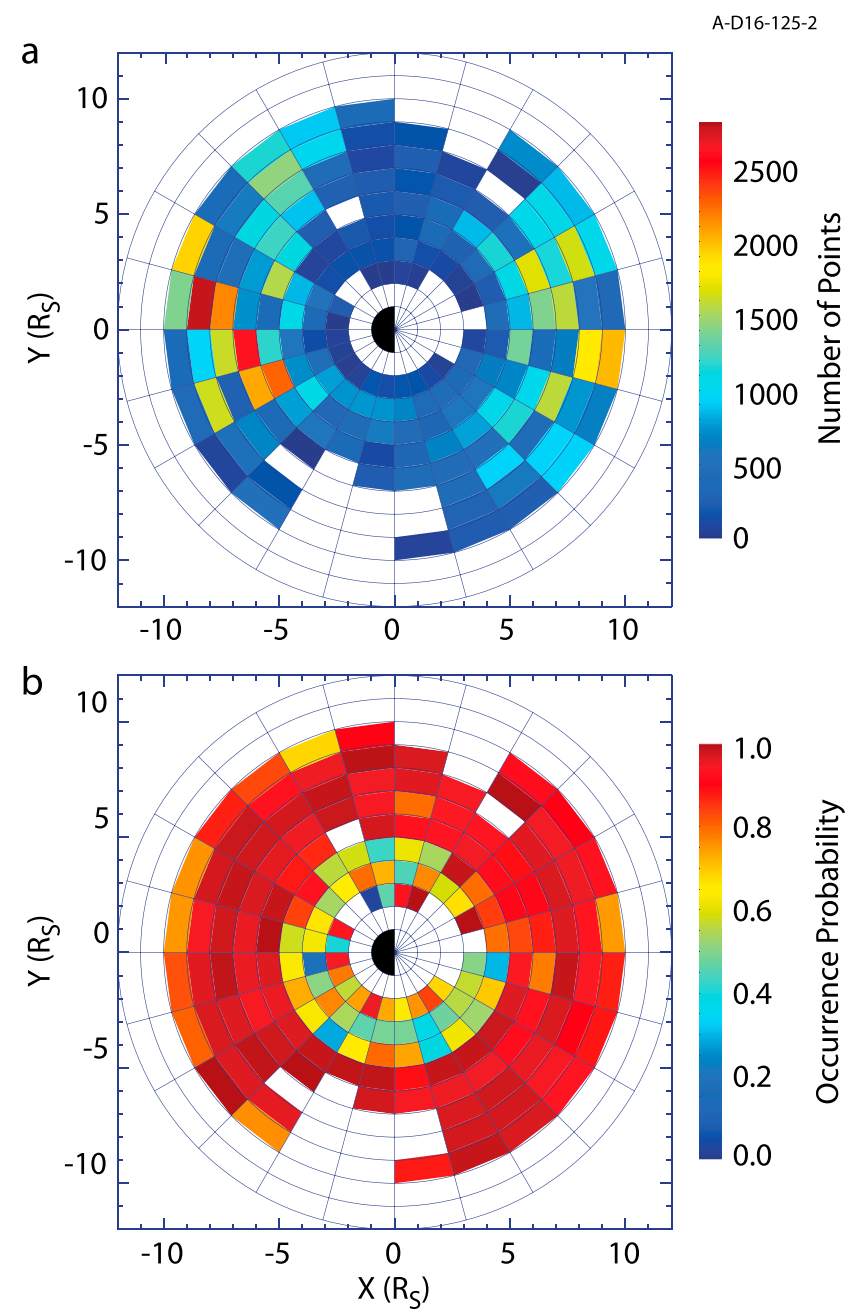

Figure 4. (a) Equatorial plane color contour for the fundamental band (3/2). The number of points within each bin is color coded. For these plots each bin is limited to $|\lambda|<5^{\circ}$. (b) Occurrence probability is color coded. ECH is observed almost all the time in the equatorial plane over the range from $\sim 5$ $R_{S}<r<10 R_{s}$, falling off within the Enceladus torus region. times $2005 / 26602: 00$ to $2005 / 267$ 18:00. The dominant emission on the spectrogram is the $\mathrm{ECH}$ emission in the fundamental and one or two much weaker harmonics. The emission is observed inside of $10 R_{s}$ and is also seen inside the Enceladus torus where, for this time period, the density is lower (note the decrease in upper hybrid frequency, $f_{\text {uh }}$ (dependent on the electron density) for $r \lesssim 3.5 R_{s}$ (Saturn radius). For this pass the ECH is seen to be more intense than the chorus emission indicated on the plot and similar in intensity to terrestrial $\mathrm{ECH}$ observations [Meredith et al., 2009]. Figure $2 b$ is another perikrone pass of a Cassini equatorial orbit with a perikrone that lies in denser plasma than the pass of Figure 2a. There is no ECH emission near perikrone, and we also note an anomalous emission region during this pass as indicated on the plot. Within such regions it is common to observe multiharmonic ECH emission that is more intense than the surrounding magnetosphere. In Figure 3 we show a higher resolution plot of anomalous emission observed on day 118 of 2010 , likely a plasma injection region showing multiple harmonics of intense emission from 07:54 to $08: 19$. Outside this isolated region of anomalous emission, ECH emission extends only to the first harmonic and at moderate intensity levels. The upper hybrid frequency is shown as determined from the digital database for the time preceding the anomalous emission region. During the anomalous emission the value $f_{\mathrm{uh}}$ is not known but may appear sporadically after the event as indicated. For the time of the anomalous emission our automated methodology dictates that we obtain the upper hybrid frequency from the plasma density model of Persoon et al. [2013], which is found to be $\sim 31 \mathrm{kHz}$, so harmonic intensities up to and including $9 / 2 f_{c e}$ would be calculated at this time. Note also that the cyclotron frequency is enhanced during the time of the event.

In this computer survey we do not distinguish ECH emission from localized plasma injections or regions of anomalous emission containing unusually high intensities and/or harmonic levels. Statistical studies of plasma injections have been conducted [cf. Chen and Hill, 2008; Kennelly et al., 2013], using personal data inspection and specific computer algorithms. Identifying these regions using algorithms for the entire Cassini data set is beyond the scope of the present study, the focus of which is ECH emission extending over a significant portion of the Cassini mission. Ye et al. [2009] and Menietti et al. [2009, 2010] have studied narrowband emission source regions which are associated with intense enhancements of the upper hybrid emission and therefore overlap the frequency range of ECH emission. Later we will investigate the possible impact that plasma injections or anomalous emission such as narrowband source regions may have on our results. 

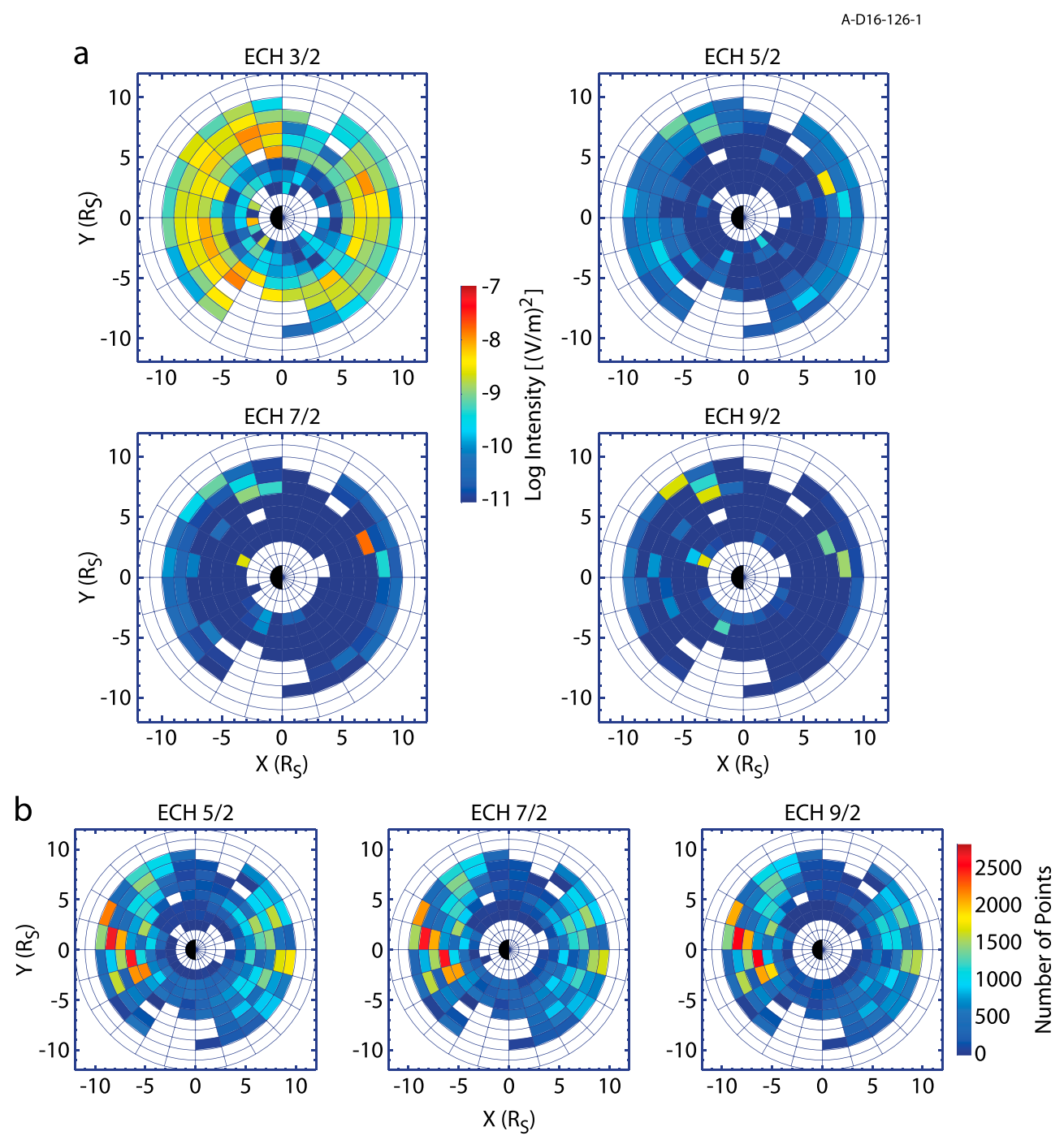

Figure 5. (a) Mean average ECH wave intensity in the equatorial plane for the fundamental and first three harmonics. The white bins are those with no observations. (b) The number of points in each bin for the ECH harmonics is color coded $\left(|\lambda|<5^{\circ}\right)$.

In Figure 4a we plot the number of points ( 1 min intensity mean values, $P_{n i}$ ) within each bin in the $x-y$ plane for the fundamental band $\left(f_{c e}<f<2 f_{c e}\right)$ of the $\mathrm{ECH}$. For these plots each bin is limited to $|\lambda|<5^{\circ}$. These numbers range from 1 to over 2500 and are absent along a band running near the dawn-dusk local time line. There were two bins with less than 10 points, while the white bins indicate no coverage by Cassini. The bins with the largest numbers are primarily on the nightside, where $\mathrm{ECH}$ emission is more probable. In Figure $4 \mathrm{~b}$ we plot the occurrence probability showing that $\mathrm{ECH}$ is observed almost all the time in the equatorial plane over the range from $\sim 5 R_{s}<r<10 R_{s}$, falling off within the Enceladus torus region. The occurrence probability for a spatial bin is defined as the total time of observations of ECH (that is emission satisfying the constraints above) divided by the total amount of time the spacecraft spent in the specified spatial bin, $T_{\text {bin }}$. We could write this as $\Sigma \Delta \tau_{i} / T_{\text {bin }}$ where the index $i$ is for each nonzero value of $P_{n i}$ within a spatial bin and frequency band, $n$. Note that, ECH emission is observed for radial distances inside the inner edge of the Enceladus torus. Figure 5a displays the mean ECH wave intensity that satisfies the constraints specified above in the equatorial plane for the fundamental and first three harmonics. The intensity levels are typically between $10^{-9}$ and $10^{-8} \mathrm{~V}^{2} / \mathrm{m}^{2}$ with a number of regions peaking at levels higher than $10^{-7} \mathrm{~V}^{2} / \mathrm{m}^{2}$. The white bins are those 

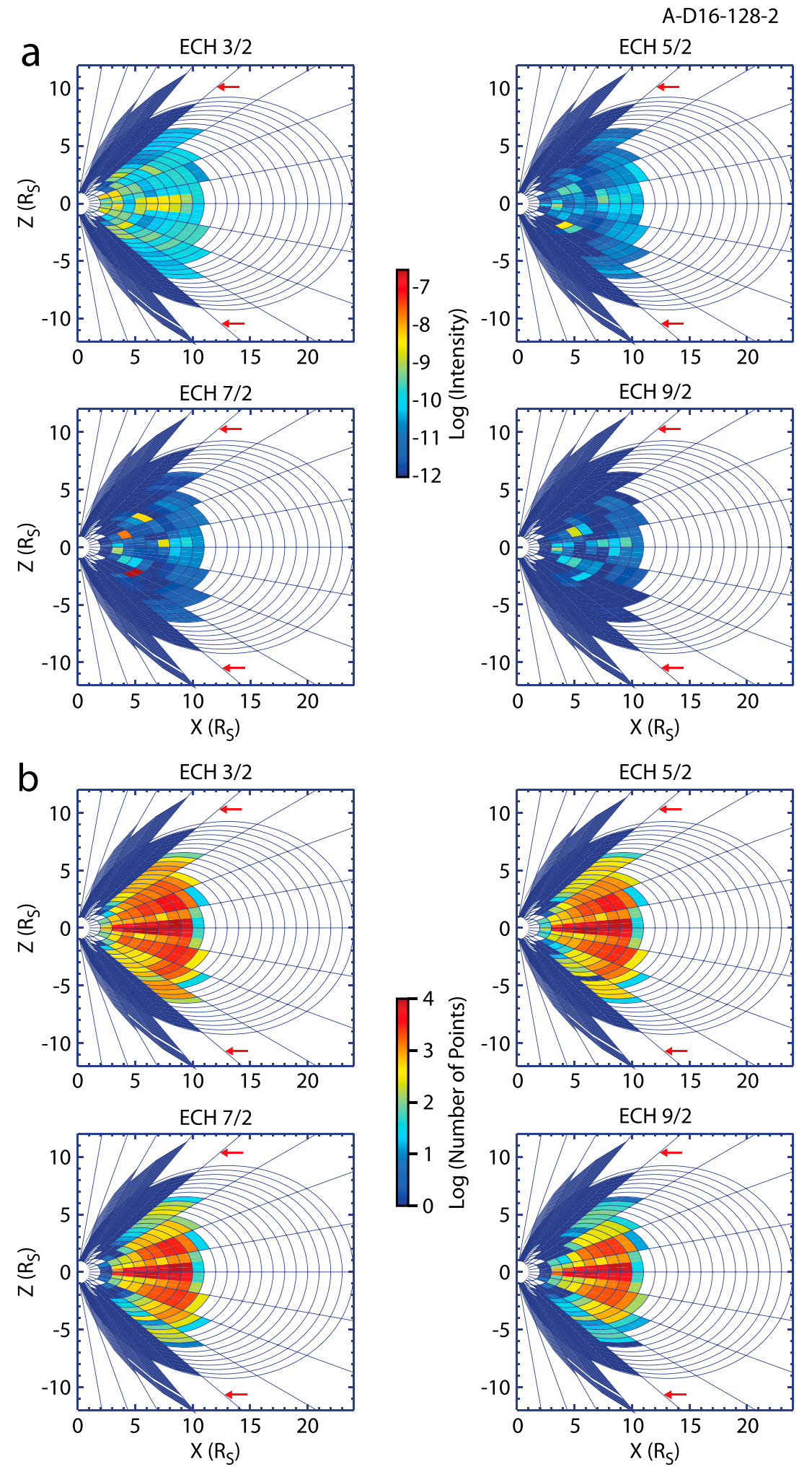

Figure 6. (a) Mean intensity versus latitude in a meridian plane for four frequency bands is shown. The data are averaged over all local times within each bin. For the fundamental band (3/2) the maximum intensity lies near the equator for $r>5$ $R_{s}$, but there are also high intensities at larger latitudes. At higher harmonics the highest intensities are only at higher latitudes. Data are for $\lambda<40^{\circ}$, indicated by the red arrow. (b) The number of points in each bin for the fundamental and harmonics of Figure $6 a$. 

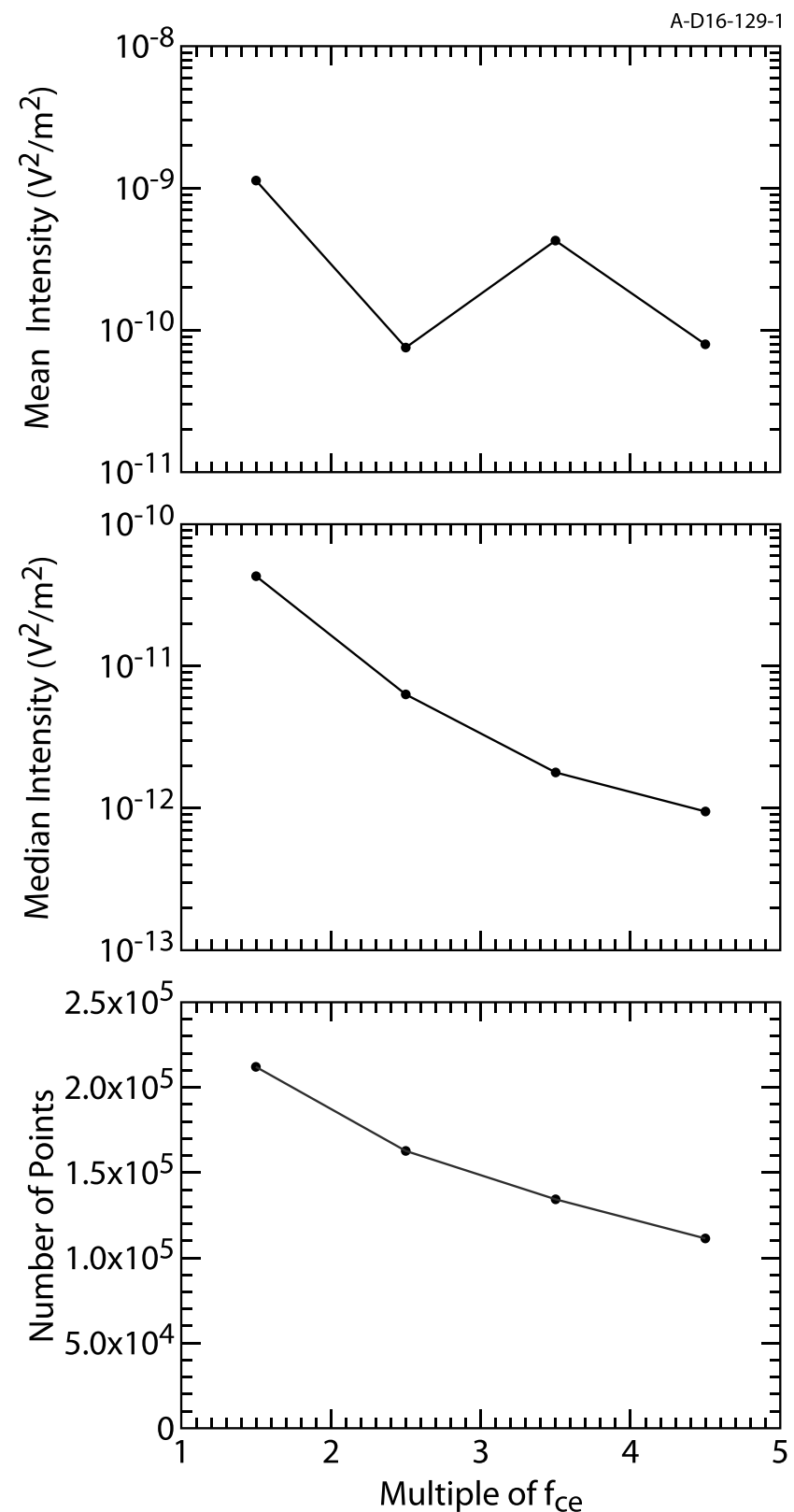

Figure 7. Mean and median intensity versus harmonic band number, independent of where the emission occurs. (top) We see that the most intense average emission is $3 / 2 f_{c e}$, with enhancements at the higher harmonics. (middle) The same results but now for the median intensity average. (bottom) The number of points for each harmonic band. with no observations. Fundamental $\mathrm{ECH}$ emission is sometimes intense at radial distances less than the inner edge of the Enceladus torus near $3 R_{s}$. Beyond $5 R_{s}$, the levels remain moderate out to $\sim 9 R_{s}$. The higher harmonics generally fall in intensity with harmonic number and show far less coverage in local time. There are sporadic enhancements in the emission at the higher harmonics which we will investigate later. Figure $5 b$ depicts the number of points in each bin for the corresponding ECH harmonics of Figure 5 a.

Mean intensity versus latitude in a meridian plane is shown for four bands in Figure 6a. Here we average the data at all local times within each bin. For the fundamental band (3/2) the maximum intensity lies near the equator for $r>5 R_{s}$, but there are also high intensities at larger latitudes. At higher harmonics the highest intensities are only at higher latitudes, which we will discuss in the next section. In Figure $6 \mathrm{~b}$ we plot the number of points (the number of $1 \mathrm{~min}$ averages, $\left.P_{n i}\right)$ for each ECH harmonic. For all of the harmonics the equatorial bins in the range $4.5<r<10$ have the most points $(>5000)$. For the higher latitudes there are still many bins in the thousands, but the number of points falls off, especially for the higher harmonics, to values less than 100 .

\section{ECH Intensity Distributions}

For the following analyses we will investigate the distribution of $\mathrm{ECH}$ intensity as a function of frequency band, $L$ shell, and latitude. This also allows us to expand the bin size and increase the number of points within each bin. We display in line plots the ECH intensity distributions of the mean and median intensity within each bin.

In Figure 7 we plot the mean and median ECH intensity that satisfies all constraints above versus harmonic band number, independent of where the emission occurs. In Figure 7 (top) we see the most intense average emission is $3 / 2 f_{c e}$, but there are increases at the higher harmonics which will be investigated later. Figure 7 (middle) displays the same results but now for the median intensity. There is a smooth decrease in intensity, and the intensity levels are about an order of magnitude lower. Figure 7 (bottom) shows the number of points ( 1 min mean or median values, $P_{n i}$ ) for each harmonic band. 

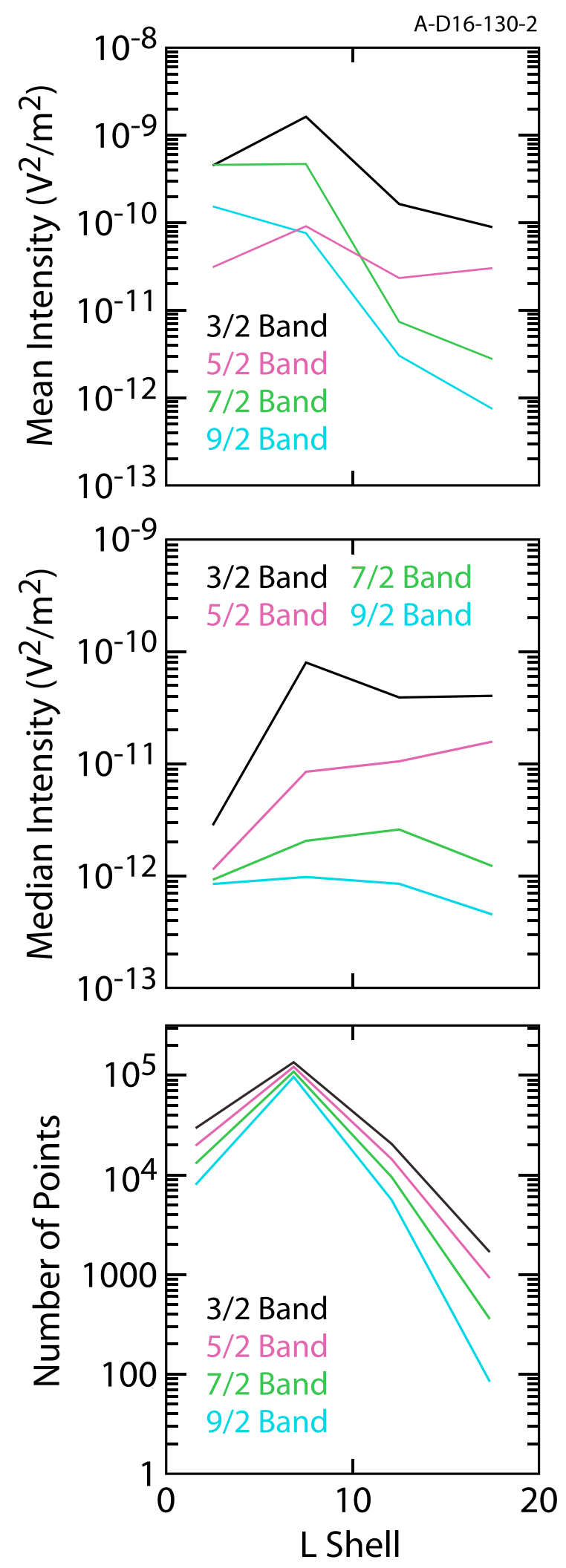

Figure 8. The (top) mean and (middle) median intensity of ECH emissions versus $L$ shell. We have averaged the intensity over each frequency band and over all local times and latitudes within bins of $L(\Delta L=5)$. (bottom) The number of points for each harmonic.
Figure 8 displays the mean (Figure 8 , top) and median (Figure 8 , middle) $\mathrm{ECH}$ intensity that satisfies all the constraints defined above versus $L$ shell. We have averaged the intensity over each frequency band and over all local times and latitudes within $\mathrm{L}$-shell bins $(\Delta \mathrm{L}=5)$. In Figure 8 (top) we see the fundamental $\mathrm{ECH}$ band emission mean intensity peaks just outside the Enceladus torus near $\mathrm{L} \sim 7$ with levels exceeding $10^{-9} \mathrm{~V}^{2} / \mathrm{m}^{2}$. The higher harmonic emissions show a more irregular (spiky) distribution. In contrast, the median values show a smoother profile about an order of magnitude lower than the mean intensity values, with peaks for higher bands between $6<\mathrm{L}<14$. Finally, Figure 8 (bottom) displays the number of points (1 min values, $\left.P_{n i}\right)$ for each harmonic.

Figures 9 displays the mean (Figures 9, column 1) and median (Figures 9, column 2) ECH intensity satisfying all constraints versus latitude for respective $L$-shell ranges $\mathrm{L}<5$ (Figures 9, row 1), $5<\mathrm{L}<10$ (Figures 9, row 2), and $\mathrm{L}>10$ (Figures 9, row 3). Figures 9 (column 3) contains the number of $1 \mathrm{~min}$ values, $P_{n i}$ per bin. For $\mathrm{L}<5$, for the lowest two bands the mean intensity increases and then decreases smoothly with latitude, while the two highest bands exhibit spikier intensity profiles. The median values shown in Figures 9 (column 2) all show much lower intensity and flatter profiles versus latitude with moderate increases at some higher latitudes. For $5<\mathrm{L}<10$ the average intensity for each band falls off from a peak near the equator but increases at intermediate latitudes with spikes at the higher harmonics. The median intensity is much lower and more ordered with decreasing intensity versus latitude except for the $9 / 2$ band. Finally, the average intensities for $L>10$, which are 

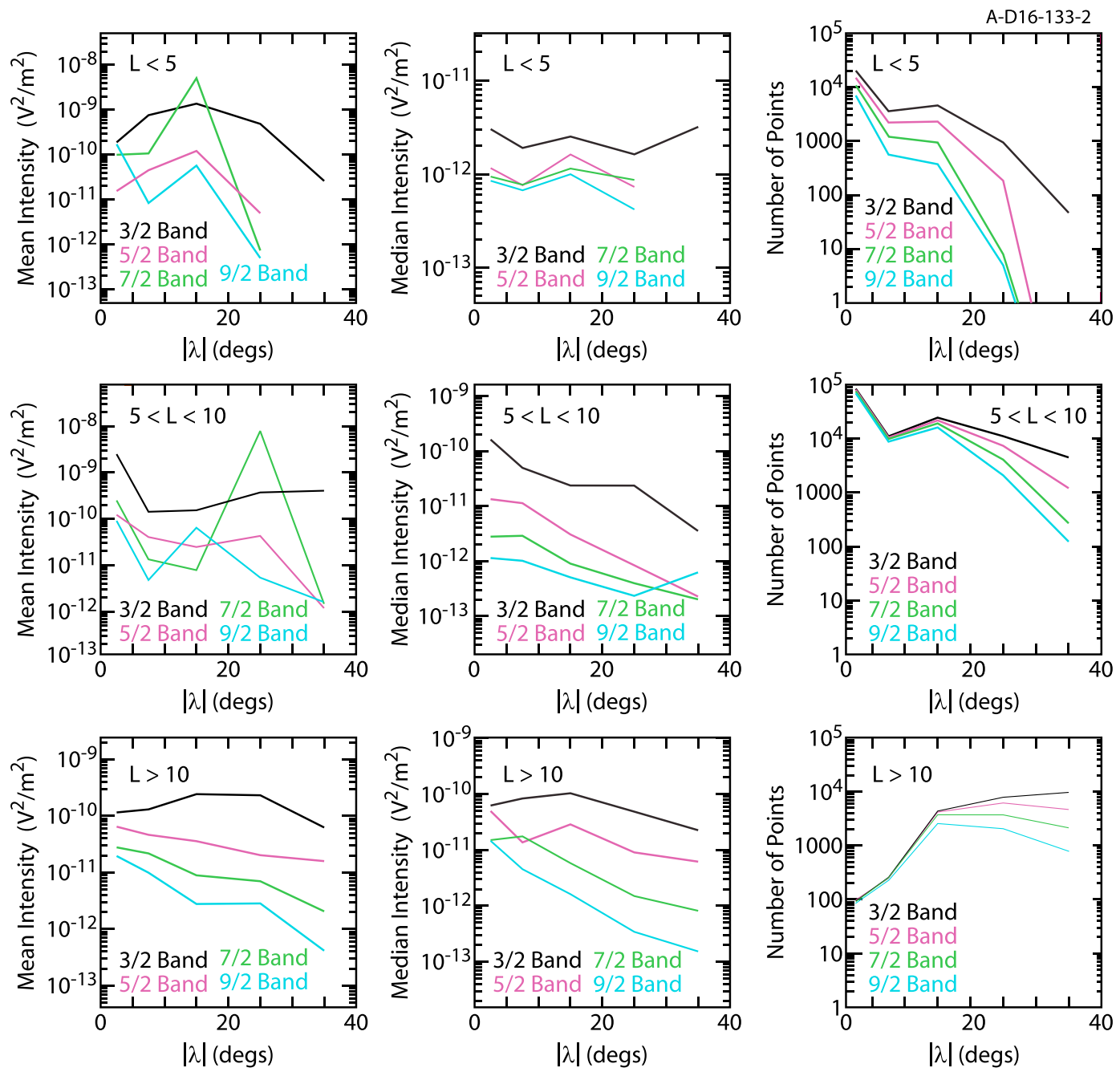

Figure 9. (column 1) Mean and (column 2) median intensity versus latitude for L-shell ranges (row 1$) \mathrm{L}<5$, (row 2 ) $5<\mathrm{L}<10$, and (row 3) $\mathrm{L}>10$. (column 3 ) The number of points per bin.

primarily from higher latitudes at smaller radial distances outside the Enceladus torus, display more order for all the harmonics with a flatter, less intense profile. The average and median intensities are similar.

\section{Injection and Anomalous Emission Regions}

A likely source of the spikes occurring in the data, particularly noticeable in Figures 8 and 9 has been determined to be isolated cases of intense injection regions (such as seen in Figure 3 ) or anomalous emission such as narrowband emission source regions. To demonstrate the impact of the injection and anomalous emission regions, we sort by magnitude the individual 1 min intensity means $\left(P_{n i}\right)$ in each of the harmonic band spatial bins of Figure 9. Each of these mean values is time-tagged, of course. We then manually examine frequencytime spectrograms of the time intervals containing the most intense emission and remove those time periods containing large plasma injections or anomalous emission. In each case this amounted to only a very small fraction of the total points per bin. We note that we did not make a comprehensive search for these regions, we eliminated only time intervals containing plasma injections or anomalous emission with the highest intensity. We replot the revised data set in Figure 10. Clearly, the most significant spikes seen in Figure 9 are now missing in Figure 10, indicating that they are due to intense plasma injection and anomalous emission regions. Relatively few numbers of anomalous emissions are responsible for largest intensity values, as evidenced by comparing the number of points $\left(P_{n i}\right)$ from column 3 of Figures 9 and 10. 

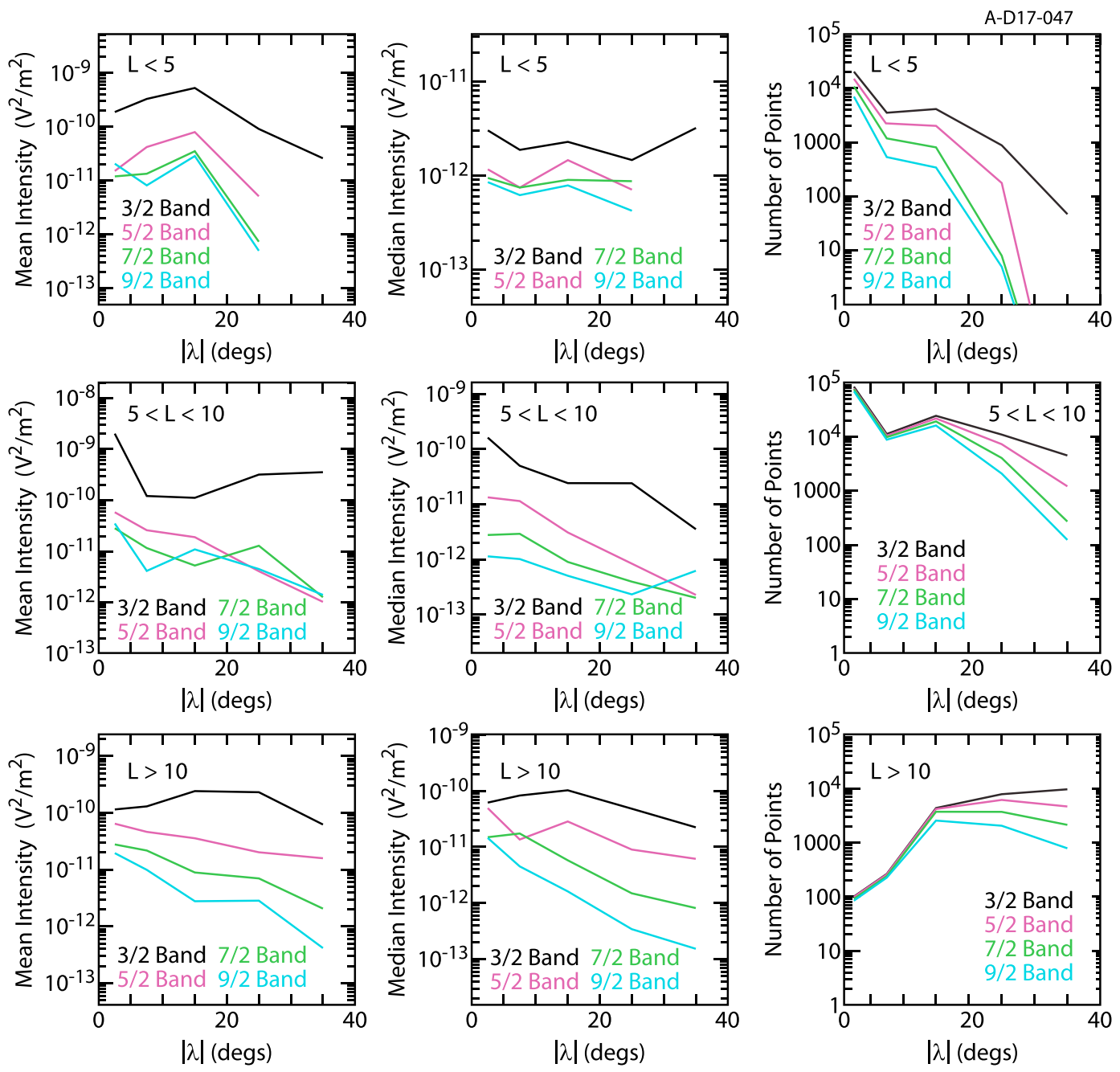

Figure 10. Same as Figure 9 except that the most intense anomalous emissions have been removed from the data.

\section{Summary and Conclusions}

ECH emissions are prevalent in the Saturn magnetosphere, with a concentration near the magnetic equator, extending from the outer Enceladus torus and falling off rapidly beyond $\sim 8 R_{s}$. Nearest the center of the Enceladus torus there is a distinct decrease in $\mathrm{ECH}$ emission. For the fundamental the distribution of intensity is relatively constant in local time but becomes far more sporadic at the harmonics, likely due to the presence of plasma injection or anomalous emission regions. These regions typically contain intense multiharmonic ECH emission [cf. Hospodarsky et al., 2008; Menietti et al., 2008], due to the enhanced ratio of $T_{h} / T_{c}$ [Rymer et al., 2008]. It is clear that there are smoother, more ordered, and weaker intensities for the median compared to the mean average, probably due to the presence of intense injection and anomalous emission regions that can extend to high $L$ shells at low radial distance and higher latitude. In particular, when the sorted averages of the bins of Figure 9 are examined and reprocessed by removing the most intense examples of localized injection regions and anomalous emissions, the spikes in Figure 9 are greatly diminished (Figure 10). The examples of localized, very intense emission are few in number, and the median values are considerably smaller than the mean values of intensity.

Thomsen et al. [2016] have reported that hot electrons ( $>$ few hundred eV) disappear in a variable L-shell range $4.7<\mathrm{L}<8.4$ consistent with the location of most observed interchange injections. This is also the region where Saturn chorus intensity emission peaks. Menietti et al. [2014] report that this occurs near $L=5.5$. In addition, the peak Z-mode intensity at Saturn is found inside the inner edge of the Enceladus 
torus at latitudes above the equator near $\sim 25^{\circ}$ [Menietti et al., 2015]. Both chorus and $Z$ mode are known to be significant sources of pitch angle and energy diffusive scattering of electrons [Glauert and Horne, 2005; Gu et al., 2013]. Finally, the peak ECH wave intensity is here found to lie at $L \sim 7.5$ for the fundamental near the equator and at higher latitudes for $r<4 R_{s}$. Since all of these waves can scatter electrons with energies in the range few hundred $\mathrm{eV}$ to tens of $\mathrm{keV}$, these findings suggest that wave particle interactions may be a likely significant reason for the observed inner edge of the suprathermal electron boundary observed by Cassini.

The importance of ECH emissions to overall magnetospheric dynamics and electron scattering in the terrestrial magnetosphere is significant as discussed in section 1, and is likely similarly significant at Saturn. These waves are known to scatter electrons into the loss cone, especially at higher latitude and lower electron energy, and thus may play an important role in the overall energy balance between energization and loss of electrons at Saturn. The intensity levels of Saturn ECH waves are comparable to and sometimes larger than those typically observed at Earth and occur in a similar frequency range [Meredith et al., 2009]. The results presented here will be important in the diffusive modeling efforts at Saturn, as has been shown at Earth by Horne et al. [2003] and other authors cited earlier.

\section{Acknowledgments}

We wish to thank J. Barnholdt and K. Kurth for administrative assistance and J. Chrisinger for much help with the figures. J.D.M. acknowledges support from JPL contract 1415150 and NASA grants NNX11AM36G and NNX16A147G. Cassini RPWS data including Langmuir Probe measurements are archived in calibrated, full resolution at the NASA Planetary Data System website: http:// pds.nasa.gov/ds-view/pds/viewDataset. jsp?dsid=CO-V/E/J/S/SS-RPWS-3-RDRLRFULL-V1.0, or contact the principal investigator. The Meudon Cassini RPWS/HFR database is found at http:// www.lesia.obspm.fr/kronos/data. From this database we accessed level 2 products, "n2," and for two-antenna and three-antenna modes we accessed the "n3d" and "n3b" data, respectively.

\section{References}

Ashour-Abdalla, M., and C. F. Kennel (1978), Nonconvective and convective electron cyclotron harmonic instabilities, J. Geophys. Res., 83, 1531-1543, doi:10.1029/JA083iA04p01531.

Bevington, P. R. (1969), Data Reduction and Error Analysis for the Physical Sciences, pp. 12-13, McGraw-Hill, New York.

Cecconi, B., and P. Zarka (2005), Direction finding and antenna calibration through analytical inversion of radio measurements performed using a system of two or three electric dipole antennas on a three-axis stabilized spacecraft, Radio Sci., 40, RS3003, doi:10.1029/ 2004RS003070.

Chen, Y., and T. W. Hill (2008), Statistical analysis of injection/dispersion events in Saturn's inner magnetosphere, J. Geophys. Res., 113, A07215, doi:10.1029/2008JA013166.

Dougherty, M. K., et al. (2004), The Cassini magnetic field investigation, Space Sci. Rev., 114, 331-282, doi:10.1007/s11214-004-1432-2.

Fischer, G., B. Cecconi, L. Lamy, S.-Y. Ye, U. Taubenschuss, W. Macher, P. Zarka, W. S. Kurth, and D. A. Gurnett (2009), Elliptical polarization of Saturn kilometric radiation observed from high latitudes, J. Geophys. Res., 114, A08216, doi:10.1029/2009JA014176.

Glauert, S. A., and R. B. Horne (2005), Calculation of pitch angle and energy diffusion coefficients with the PADIE code, J. Geophys. Res., 110, A04206, doi:10.1029/2004JA010851.

Gurnett, D. A., et al. (2004), The Cassini radio and plasma wave investigation, Space Sci. Rev., 114, 395-463, doi:10.1007/s11214-004-1434-0. Gurnett, D. A., et al. (2005), Radio and plasma wave observations at Saturn form Cassini's approach and first orbit, Science, 307(5713), 1255-1259, doi:10.1126/science.1105356.

Gu, X., R. M. Thorne, B. Ni, and S.-Y. Ye (2013), Resonant diffusion of energetic electrons by narrowband Z mode waves in Saturn's inner magnetosphere, Geophys. Res. Lett., 40, 255-261, doi:10.1029/2012GL054330.

Hill, T. W., A. J. Dessler, and L. J. Maher (1981), Corotating magnetospheric convection, J. Geophys. Res., 86, 9020-9028, doi:10.1029/ JA086iA11p09020.

Hill, T. W., A. M. Rymer, J. L. Burch, F. J. Crary, D. T. Young, M. F. Thomsen, D. Delapp, N. André, A. J. Coates, and G. R. Lewis (2005), Evidence for rotationally driven plasma transport in Saturn's magnetosphere, Geophys. Res. Lett., 32, L14S10, doi:10.1029/2005GL022620.

Horne, R. B., and R. M. Thorne (2000), Electron pitch angle diffusion by electrostatic electron cyclotron harmonic waves: The origin of pancake distributions, J. Geophys. Res., 105, 5391-5402, doi:10.1029/1999JA900447.

Horne, R. B., R. M. Thorne, N. P. Meredith, and R. R. Anderson (2003), Diffuse auroral electron scattering by electron cyclotron harmonic and whistler mode waves during an isolated substorm, J. Geophys. Res., 108(A7), 1290, doi:10.1029/2002JA009736.

Horne, R. B., R. M. Thorne, S. A. Glauert, J. D. Menietti, Y. Y. Shprits, and D. A. Gurnett (2008), Gyro-resonant electron acceleration at Jupiter, Nat. Phys., 4(4), 301-304, doi:10.1038/nphys897.

Hospodarsky, G. B., T. F. Averkamp, W. S. Kurth, D. A. Gurnett, J. D. Menietti, O. Santolik, and M. K. Dougherty (2008), Observations of chorus at Saturn using the Cassini Radio and Plasma Wave Science instrument, J. Geophys. Res., 113, A12206, doi:10.1029/2008JA013237.

Kennelly, T. J., J. S. Leisner, G. B. Hospodarsky, and D. A. Gurnett (2013), Ordering of injection events within Saturnian SLS longitude and local time, J. Geophys. Res. Space Physics, 118, 832-838, doi:10.1002/jgra.50152.

Kurth, W. S., D. D. Barbosa, D. A. Gurnett, and F. L. Scarf (1980), Electrostatic waves in the Jovian magnetosphere, Geophys. Res. Lett., 7, 57-60, doi:10.1029/GL007i001p00057.

Koons, H. C., and J. L. Roderer (1990), A survey of equatorial magnetospheric wave activity between 5 and 8 RE, Planet. Space Sci., 38 , 1335-1341, doi:10.1016/0032-0633(90)90136-E.

Menietti, J. D., O. Santolik, A. M. Rymer, G. B. Hospodarsky, A. M. Persoon, D. A. Gurnett, A. J. Coates, and D. T. Young (2008), Analysis of plasma waves observed within local plasma injections seen in Saturn's magnetosphere, J. Geophys. Res., 113, A05213, doi:10.1029/2007JA012856.

Menietti, J. D., S.-Y. Ye, P. H. Yoon, O. Santolik, A. M. Rymer, D. A. Gurnett, and A. J. Coates (2009), Analysis of narrowband emission observed in the Saturn magnetosphere, J. Geophys. Res., 114, A06206, doi:10.1029/2008JA013982.

Menietti, J. D., P. H. Yoon, Y. Sheng-Yi, B. Cecconi, and A. M. Rymer (2010), Source mechanism of Saturn narrowband emission, Ann. Geophys., 28, 1013-1021, doi:10.5194/angeo-28-1013-2010.

Menietti, J. D., Y. Y. Shprits, R. B. Horne, E. E. Woodfield, G. B. Hospodarsky, and D. A. Gurnett (2012), Chorus, ECH, and Z mode emissions observed at Jupiter and Saturn and possible electron acceleration, J. Geophys. Res., 117, A12214, doi:10.1029/2012JA018187.

Menietti, J. D., T. F. Averkamp, J. B. Groene, R. B. Horne, Y. Y. Shprits, E. E. Woodfield, G. B. Hospodarsky, and D. A. Gurnett (2014), Survey analysis of chorus intensity at Saturn, J. Geophys. Res. Space Physics, 119, 8415-8425, doi:10.1002/2014JA020523.

Menietti, J. D., T. F. Averkamp, S.-Y. Ye, R. B. Horne, E. E. Woodfield, Y. Y. Shprits, D. A. Gurnett, A. M. Persoon, and J.-E. Wahlund (2015), Survey of Saturn Z-mode emission, J. Geophys. Res. Space Physics, 120, 6176-6187, doi:10.1002/2015JA021426. 
Meredith, N. P., R. B. Horne, R. M. Thorne, and R. R. Anderson (2009), Survey of upper band chorus and ECH waves: Implications of the diffuse aurora, J. Geophys. Res., 114, A07218, doi:10.1029/2009JA014230.

Moncuquet, M., A. Lecacheux, N. Meyer-Vernet, B. Cecconi, and W. S. Kurth (2005), Quasi thermal noise spectroscopy in the inner magnetosphere of Saturn with Cassini/RPWS: Electron temperatures and density, Geophys. Res. Lett., 32, L20S02, doi:10.1029/2005GL022508.

Morooka, M. W., et al. (2009), The electron density of Saturn's magnetosphere, Ann. Geophys., 27, 2971-2991, doi:10.5194/ angeo-27-2971-2009.

Ni, B., R. Thorne, J. Liang, V. Anelopoulos, C. Cully, W. Li, X. Zhang, M. Hartinger, O. Le Contel, and A. Roux (2011), Global distribution of electrostatic electron cyclotron harmonic waves observed on THEMIS, Geophys. Res. Lett., 38, L17105, doi:10.1029/2011GL048793.

Persoon, A. M., et al. (2009), A diffusive equilibrium model for the plasma density in Saturn's magnetosphere, J. Geophys., Res., 114, A04211 doi:10.1029/2008JA013912.

Persoon, A. M. D., A. Gurnett, J. S. Leisner, W. S. Kurth, J. B. Groene, and J. B. Faden (2013), The plasma density distribution in the inner region of Saturn's magnetosphere, J. Geophys. Res. Space Physics, 118, 2970-2974, doi:10.1002/jgra.50182.

Persoon, A. M., D. A. Gurnet, W. S. Kurth, J. B. Groene, and J. B. Faden (2015), Evidence for a seasonally dependent ring plasma in the region between Saturn's A Ring and Enceladus' orbit, J. Geophys., Res. Space Physics, 120, 6276-6285, doi:10.1002/2015JA021180.

Pontius, D. H., Jr., T. W. Hill, and M. E. Rassbach (1986), Steady state plasma transport in a corotation-dominated magnetosphere, Geophys. Res. Lett., 13, 1097-1100, doi:10.1029/GL013i011p01097.

Reeves, G. D., et al. (2013), Electron acceleration in the heart of the Van Allen radiation belts, Science, 341, doi:10.1126/science.1237743.

Rymer, A. M., B. H. Mauk, T. W. Hill, C. Paranicas, D. G. Mitchell, A. J. Coates, and D. T. Young (2008), Electron circulation in Saturn's magnetosphere, J. Geophys. Res., 113, A01201, doi:10.1029/2007JA012589.

Shprits, Y. Y., D. Subbotin, and N. Ni (2009), Evolution of electron fluxes in the outer radiation belt computed with the VERB code, J. Geophys. Res., 114, A11209, doi:10.1029/2008JA013784.

Shprits, Y. Y., J. D. Menietti, X. Gu, K.-C. Kim, and R. B. Horne (2012), Gyro-resonant interactions between the radiation belt electrons and whistler mode chorus waves in the radiation environments of Earth, Jupiter, and Saturn, a comparative study, J. Geophys. Res., 117, A11216, doi:10.1029/2012JA018031.

Tao, X., R. M. Thorne, R. B. Horne, S. Grimald, C. S. Arridge, G. B. Hospodarsky, D. A. Gurnett, A. J. Coates, and F. J. Crary (2010), Excitation of electron cyclotron harmonic waves in the inner Saturn magnetosphere within local plasma injections, J. Geophys. Res., 115, A12204, doi:10.1029/2010JA015598.

Thomsen, M. F., A. J. Coates, E. Roussos, R. J. Wilson, K. C. Hansen, and G. R. Lewis (2016), Suprathermal electron penetration into the inner magnetosphere of Saturn, J. Geophys. Res. Space Physics, 121, 5436-5448, doi:10.1002/2016JA022692.

Thorne, R. M., B. Ni, X. Tao, R. B. Horne, and N. P. Meredith (2010), Scattering by chorus waves as the dominant cause of diffuse auroral precipitation, Nature, 467, 943-946, doi:10.1038/nature09467.

Wahlund, J.-E., et al. (2005), The inner magnetosphere of Saturn: Cassini RPWS cold plasma results from the first encounter, Geophys. Res. Lett., 32, L20509, doi:10.1029/2005GL022699.

Woodfield, E. E., R. B. Horne, S. A. Glauert, J. D. Menietti, and Y. Y. Shprits (2014), The origin of Jupiter's outer radiation belt, J. Geophys. Res. Space Physics, 119, 3490-3502, doi:10.1002/2014JA019891.

Ye, S.-Y., D. A. Gurnett, G. Fischer, B. Cecconi, J. D. Menietti, W. S. Kurth, Z. Wang, G. B. Hospodarsky, P. Zarka, and A. Lecacheux (2009), Source locations of narrowband radio emissions detected at Saturn, J. Geophys. Res., 114, A06219, doi:10.1029/2008JA013855.

Ye, S.-Y., J. D. Menietti, G. Fischer, Z. Wang, B. Cecconi, D. A. Gurnett, and W. S. Kurth (2010), Z mode waves as the source of Saturn narrowband radio emissions, J. Geophys. Res., 115, A08228, doi:10.1029/2009JA015167.

Yoon, P. H., A. T. Weatherwax, and T. J. Rosenberg (1998), On the generation of auroral radio emissions at harmonics of the lower ionospheric electron cyclotron frequency: $X, O$ and $Z$ mode maser calculations, J. Geophys. Res., 103(A3), 4071-4078, doi:10.1029/97JA03526.

Zarka, P., B. Cecconi, and W. S. Kurth (2004), Jupiter's low-frequency radio spectrum from Cassini/Radio and Plasma Wave Science (RPWS) absolute flux density measurements, J. Geophys. Res., 109, A09S15, doi:10.1029/2003JA010260. 